\title{
Intelligent Control for Drag Reduction on the X-48B Vehicle
}

\author{
Brian Joseph Griffin*, Nelson A. Brown ${ }^{\dagger}$, and Seung Y. Yoo \\ NASA Dryden Flight Research Center, Edwards, California, 93523
}

\begin{abstract}
This paper focuses on the development of an intelligent control technology for in-flight drag reduction. The system is integrated with and demonstrated on the full X-48B nonlinear simulation. The intelligent control system utilizes a peak-seeking control method implemented with a time-varying Kalman filter. Performance functional coordinate and magnitude measurements, or independent and dependent parameters respectively, are used by the Kalman filter to provide the system with gradient estimates of the designed performance function which is used to drive the system toward a local minimum in a steepestdescent approach. To ensure ease of integration and algorithm performance, a single-input single-output approach was chosen. The framework, specific implementation considerations, simulation results, and flight feasibility issues related to this platform are discussed.
\end{abstract}

\section{Introduction}

This paper focuses on an intelligent control for performance (ICP) technology for drag reduction on the $\mathrm{X}-48 \mathrm{~B}$ vehicle which is integrated as an outer-loop system and is demonstrated on the full $\mathrm{X}$-48B non-linear simulation. This simulation produces the actual flight code flown onboard the vehicle. Unique characteristics of this implementation are the vehicle platform itself and the challenges it presents as well as the time-varying Kalman filter driven peak-seeking control (PSC) algorithm. In addition to discussing the implementation considerations on this platform and the presenting simulation results, problems are presented that could impact the feasibility of flight-testing this technology. A brief background is presented below, followed by a more detailed vehicle description.

The Environmentally Responsible Aviation (ERA) project is a project under the newly created Integrated Systems Research Program (ISRP), which focuses on maturing and integrating NextGen technologies into major vehicle operational systems and subsystems. The primary goal of the ERA project is to select vehicle concepts and technologies that can simultaneously reduce fuel burn, noise and emissions. ${ }^{1}$ One such vehicle configuration proving the potential of increased performance in each of these areas is the Hybrid Wing Body (HWB) configuration.

The HWB aircraft configuration represents a novel and significant design departure from the traditional "tube-and-wing" transport aircraft configuration. The HWB configuration provides minimal distinction between the wing, fuselage, and tail sections of the vehicle. This configuration may closely resemble a flying wing configuration, however, the HWB configuration concentrates significantly more volume in the center section of the aircraft as opposed to a traditional flying wing. This configuration holds promise for remarkably improved subsonic transport efficiency over traditional transport aircraft such as high internal volume, aerodynamic and structural efficiency, and lower noise. Design studies have shown that this configuration will have lower takeoff weight and lower fuel burn compared to conventional designs given the same mission. ${ }^{2}$ Performance advantages such as the reduced fuel burn can be made even greater by the implementation of new intelligent control methods.

Previous work at the NASA Dryden Flight Research Center (DFRC) (Edwards, California) used real-time performance optimization techniques employing peak-seeking control (PSC) methods to improve performance on various vehicle configurations. ${ }^{3-12}$ The demonstrated technology was transitioned to measurement-based

\footnotetext{
*Aerospace Engineer, Flight Controls and Dynamics Branch, P.O. Box 273, MS 4840D, brian.j.griffin@nasa.gov.

$\dagger$ Aerospace Engineer, Flight Controls and Dynamics Branch, P.O. Box 273, MS 4840D, nelson.a.brown@nasa.gov.

¥Aerospace Engineer, Flight Controls and Dynamics Branch, P.O. Box 273, MS 4840D, seung.y.yoo@nasa.gov.
} 
adaptive optimal control for performance improvement applied to tube-and-wing transport configurations utilizing redundant control effectors. Modes of operation included minimization of fuel consumption and maximizing velocity. Compensation is achieved with continuous feedback of parameter measurements influencing the optimization objective resulting in optimized control effector positions. In this work PSC is used to search for a minimum drag configuration at a given flight condition. Presented are unique challenges resulting from the HWB vehicle configuration itself such as maintaining pitch trim while implementing an outer-loop system on top of a closed-loop feedback control system within the allocation scheme of the vehicle. In addition, the new PSC method presents integration challenges as well.

\section{Vehicle Description}

The X-48B vehicle, see Fig. (1), is an 8.5-percent dynamically-scaled HWB constructed for The Boeing Company (Boeing Phantom Works, Huntington Beach, California) by Cranfield Aerospace Ltd. (Bedford, United Kingdom). The remotely piloted vehicle was developed as a technology demonstrator to investigate the flight characteristics of the HWB design. The X-48B has a wingspan of $20.4 \mathrm{ft}$ and a maximum fueled weight of $525 \mathrm{lb}$. Three JetCat USA (Paso Robles, California) P200 turbojet engines, each capable of 54 $\mathrm{lb}$ of thrust, power the aircraft. The X-48B has two fixed leading-edge, ground-configurable slat positions, extended and retracted, and 20 control surfaces including a winglet rudder and two clamshells on each side. These surfaces are driven using a closed-loop feedback control system according to the allocation scheme shown in Fig (2). Sensed control surface position feedbacks as well as current engine core speeds are sensed and used in the intelligent control system presented in this work. In addition, the vehicle is equipped with both auto-pilot and auto-throttle systems which are utilized in the intelligent control system design presented.

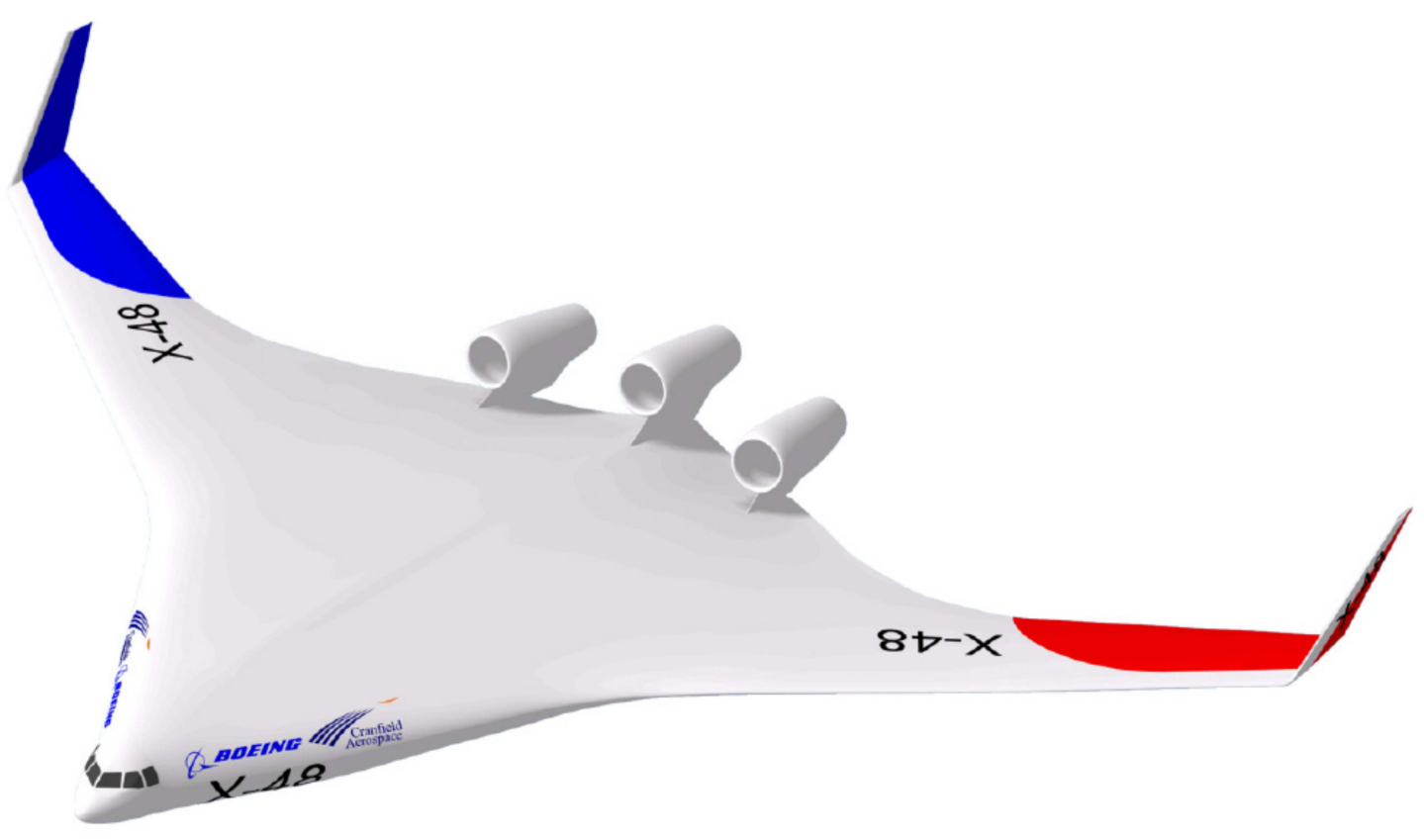

Figure 1. The configuration of the $\mathrm{X}-48 \mathrm{~B}$ vehicle. 


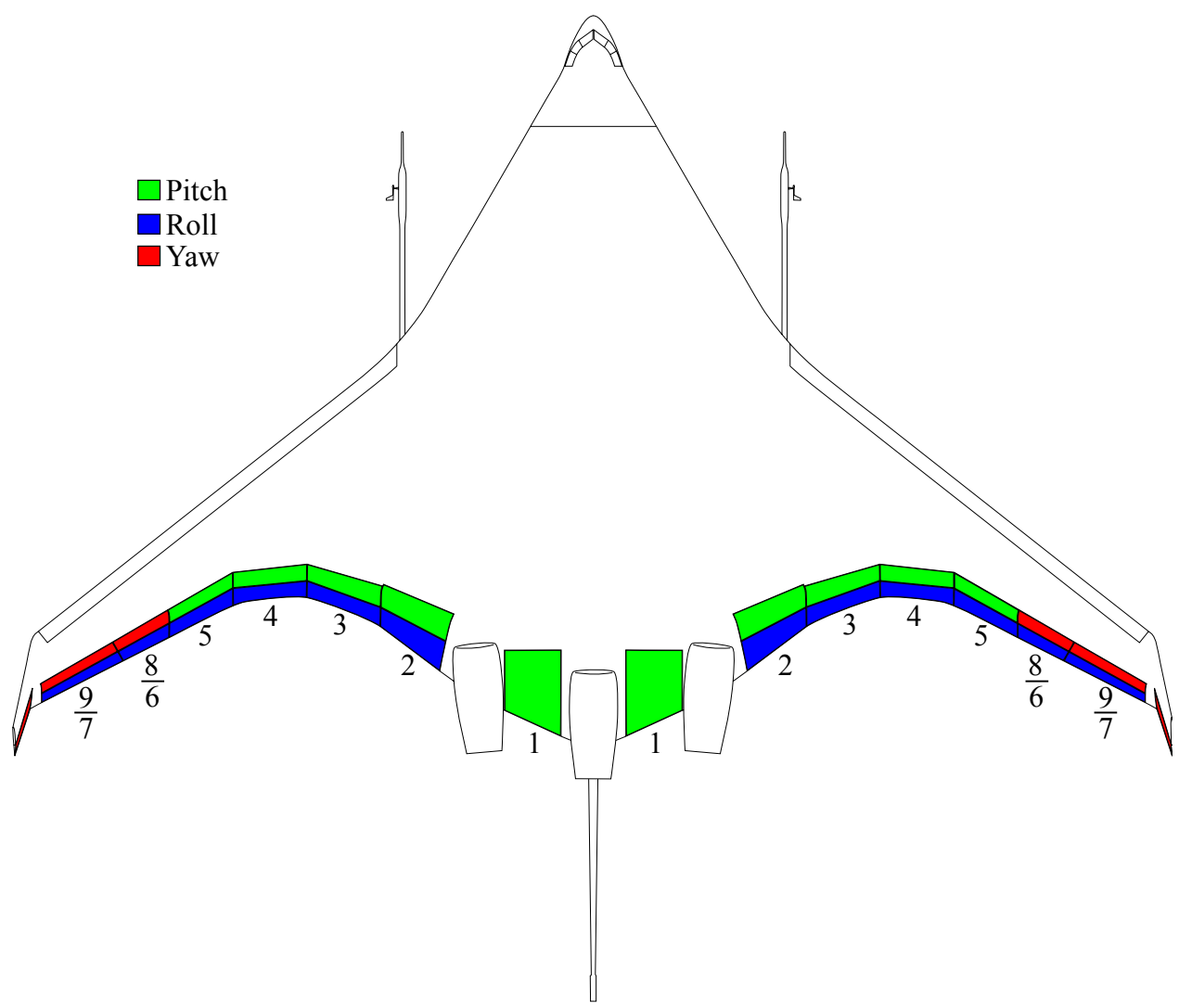

Figure 2. The baseline allocation scheme of the $\mathrm{X}-48 \mathrm{~B}$ vehicle.

\section{Framework}

The PSC algorithm in this work is adapted from previous work and is shown below. ${ }^{13}$ In the most general terms, the goal of the intelligent control design using PSC methods is to control a plant in such a way as to ensure desired behavior in addition to driving some defined output, $\boldsymbol{x}(t)$ of the plant $P$, to the extremum of a performance function, $f(\boldsymbol{x}(t))$. Figure 3 depicts the peak-seeking control interconnection.

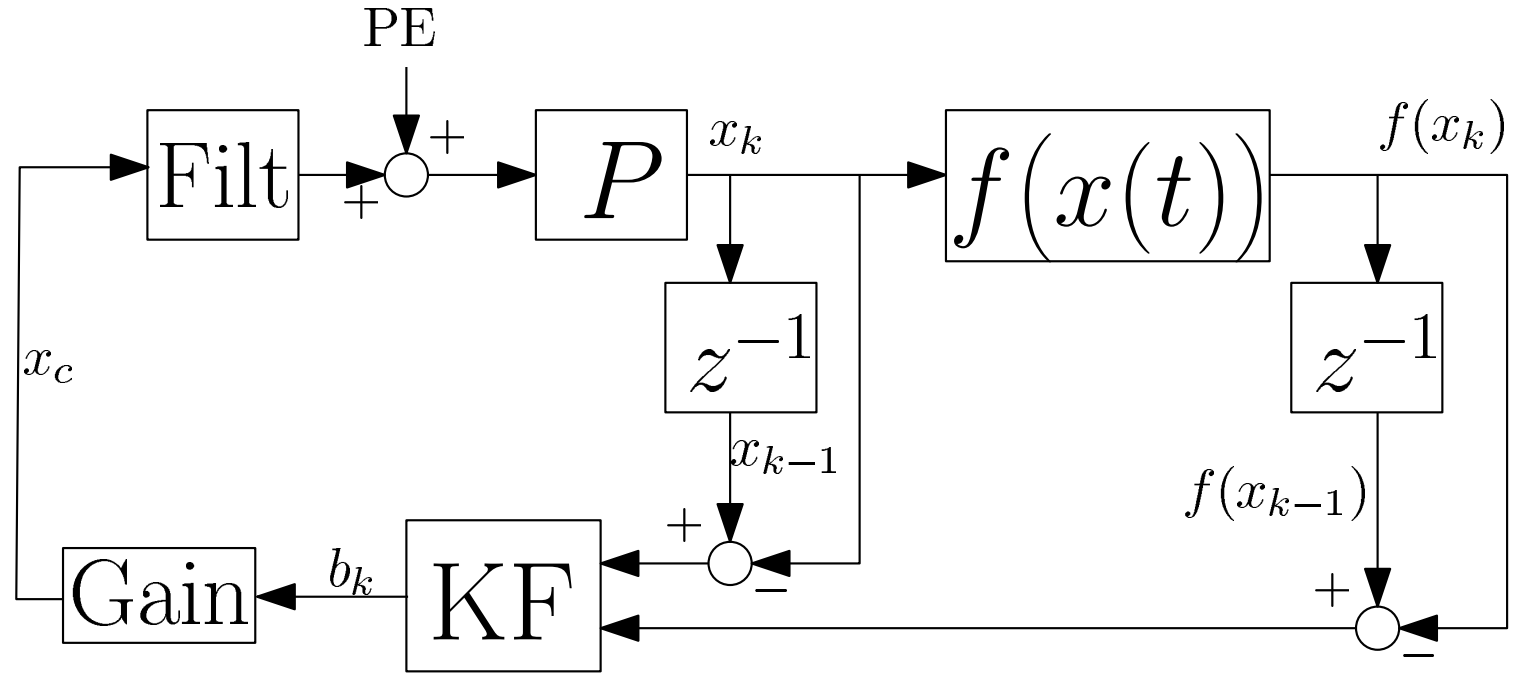

Figure 3. Peak-seeking framework interconnection. 
Here, the performance function independent parameter, $\boldsymbol{x}(t)$, is one or more outputs of the plant, $P$. The PSC method provides an outer-loop optimization of the defined performance function and it assumes inner-loop control of the plant is provided. At each iteration of the optimization, differences are calculated between the previous and current output, $\boldsymbol{x}_{k-1}-\boldsymbol{x}_{k}$, and the previous and current performance function magnitude, $f\left(\boldsymbol{x}_{k-1}\right)-f\left(\boldsymbol{x}_{k}\right)$. From these, a linear time-varying Kalman filter (KF) provides estimates of the current gradient, $\boldsymbol{b}_{k}$ of the performance function. In this application a constant gain multiplier is used to drive the plant in a steepest-descent command approach. This is then further filtered to smooth the commands to avoid large step inputs which can produce undesired actuator movements as well as drive the plant off trim. In the approach taken in this work, the performance function is defined in terms of a single independent parameter and in practice, an initial command changing this parameter will initiate movement of the system along the performance function allowing gradient estimation. This is all that's required, avoiding the necessity to provide any defined persistent excitation (PE) to the system.

\section{A. Kalman Filter Implementation}

The heart of this peak-seeking control scheme is the use of the linear time-varying Kalman filter which uses position measurements directly and whose states contain the current gradient estimates. A Taylor series expansion of the performance function $f(\boldsymbol{x})$ about $\boldsymbol{x}_{k}$ is given by Eq. (1):

$$
f(\boldsymbol{x}) \approx f\left(\boldsymbol{x}_{k}\right)+\boldsymbol{b}_{k}^{\top}\left(\boldsymbol{x}-\boldsymbol{x}_{k}\right)+O\left(\boldsymbol{x}-\boldsymbol{x}_{k}\right)
$$

where $O(\cdot)$ represents higher-order terms. Assuming the performance function can be sufficiently treated as linear at any position, at each iteration the difference between the previous and current performance function magnitude can then be approximated as shown in Eq. (2):

$$
\Delta f_{k}=\boldsymbol{b}_{k}^{\top} \Delta \boldsymbol{x}_{k}
$$

where $\Delta f_{k}=f\left(\boldsymbol{x}_{k-1}\right)-f\left(\boldsymbol{x}_{k}\right)$ and $\Delta \boldsymbol{x}_{k}=\boldsymbol{x}_{k-1}-\boldsymbol{x}_{k}$. To remain consistent with the approach taken in this work, the implementation will be restricted to a single-dimension case. Under these conditions, Eq. (2) can be rewritten simply as Eq. (3):

$$
\Delta f_{k}=\Delta x_{k} b_{k}
$$

Expecting the gradient to change as a function of position, possibly noisy measurements, and time-varying $\Delta x_{k}$, a linear time-varying Kalman filter is an appropriate choice of estimator. The state becomes the gradient to be estimated given as $\zeta_{k}=b_{k}$ and the measurement equation of the linear time-varying Kalman filter is given by Eq. (4):

$$
\Delta f_{k}=H_{k} \zeta_{k}+v_{k}
$$

where $H_{k}=\Delta x_{k}$ and $v_{k}$ represents a zero-mean Gaussian white-noise process with variance $V_{k}$. Given the unknown true dependance of the performance function on the plant output $x_{k}$, the state is modeled as a Brownian noise process and the linear time-varying Kalman filter process equation is then given by Eq. (5):

$$
\zeta_{k+1}=\zeta_{k}+w_{k}
$$

where $w_{k}$ represents a zero-mean Gaussian white-noise process with variance $W_{k}$. The linear time-varying Kalman filter implementation is given from Eqs. (6a) through (6e):

$$
\begin{aligned}
K & =\hat{P}_{k} H_{k}^{\top}\left(H_{k} \hat{P}_{k} H_{k}^{\top}+V_{k}\right)^{-1} \\
\zeta_{k} & =\hat{\zeta}_{k}+K\left(\Delta f_{k}-H_{k} \hat{\zeta}_{k}\right) \\
P_{k} & =\left(I-K H_{k}\right) \hat{P}_{k} \\
\hat{\zeta}_{k+1} & =\zeta_{k} \\
\hat{P}_{k+1} & =P_{k}+W_{k}
\end{aligned}
$$

where $P_{k}$ and $\hat{P}_{(\cdot)}$ are current and predicted state covariance matrices respectively at the corresponding iterations. Similarly, $\zeta_{k}$ and $\hat{\zeta}_{(\cdot)}$ are the current and predicted Kalman filter state estimate which is the gradient of the perfomance function. The noise variances $V_{k}$ and $W_{k}$ are used as tuning parameters influencing the filters performance. 


\section{Implementation}

In the X-48B application, the PSC scheme was used to dynamically allocate the control surfaces to reduce drag in flight. The algorithm generates a surface position command in order to optimize the span-wise lift distribution at a given flight condition. The surface position command is identically sent to the upper an lower surfaces of each clamshell, on both sides of the vehicle, while the inner-loop control law moves the remaining surfaces to maintain trim. The vehicle's engine core speed (N2, measured in rpm) is averaged across the three engines, resulting in a scalar output, and is used to define the magnitude of the performance function in this application. This approach was chosen because subtle changes in the aircraft's performance must be measured and fed into the intelligent control system. Additionally, a low-noise, high-precision signal will improve the convergence time of the system and $\mathrm{N} 2 \mathrm{rpm}$ was considered the best low-noise indicator available. It is used in the simulation analysis presented in this work. At a constant altitude and airspeed, a reduction in engine rpm indicates a reduction in vehicle drag.

\section{A. Allocation}

The intelligent control system will dynamically allocate a symmetric command to the clamshell surfaces of the $\mathrm{X}-48 \mathrm{~B}$ vehicle. The commanded surfaces as well as the additional control surfaces are shown in Fig. (4).

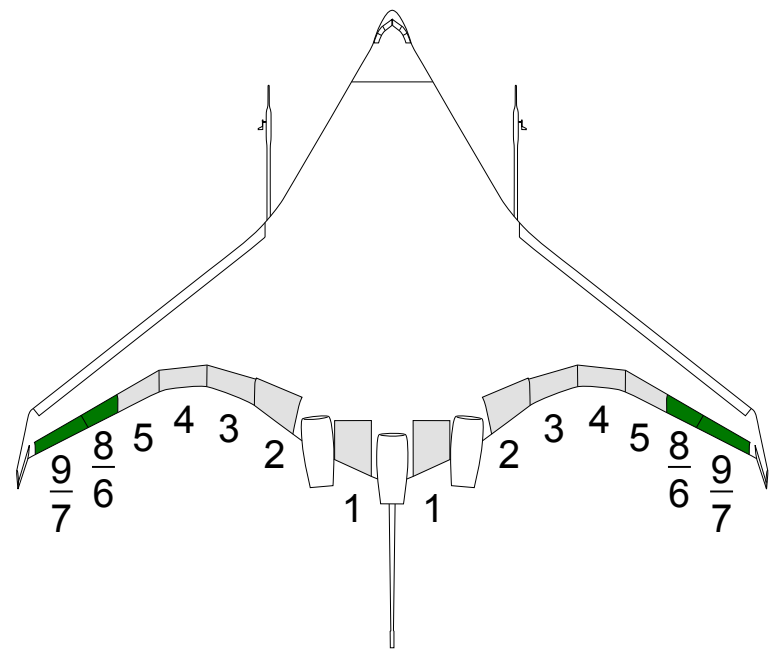

Figure 4. The green surfaces are dynamically allocated by the intelligent control system.

It was originally desired to group the clamshell surfaces (6-9) as one independent parameter of the performance function in addition to the surfaces 2-5 as a second independent parameter. In this approach, the PSC scheme would consist of two dimensions and the linear time-varying Kalman filter estimator states would include the gradients with respect to each of these parameters. In addition to increasing the number of estimates, the inner-loop control allocation scheme naturally uses sufaces 2-5 as pitch control surfaces, see Fig (2), so any offset generated by the outer-loop intelligent control system to these surfaces resulted in fighting between the two systems. Any command generated from the outer-loop intelligent control system was effectively canceled by an opposite command from the inner-loop control system to remove this disturbance. To alleviate this problem without any modification to the inner-loop control system, the approach taken was to simply use only the clamshell surfaces with the outer-loop intelligent control system. The baseline allocator will not use these surfaces to control pitch, so any pitch disturbance produced from these surfaces must be accounted for by moving surfaces 1-5, which produces the desired result and prevents fighting between the inner-loop control system and the intelligent control system.

\section{B. Measurement and Command Filtering}

Filtering and smoothing techniques were employed at both the input and output plane of the PSC system. The magnitude of the performance function was determined from the average of the three engine core rotation rates computed from the the raw sensor measurements. To avoid noisy measurement data from 
driving the PSC algorithm and potentially degrading the convergence time and performance, second-order low-pass filters were used.

The outer-loop optimization ran much slower than the inner-loop control system. This was due to the transients and settling time characteristics of the engine response from a throttle setting change. In the simulation results presented in this work, the vehicle's control system is running at $200 \mathrm{~Hz}$ whereas the outerloop intelligent control system optimization is running much slower at $0.05 \mathrm{~Hz}$. This means new measurement data is provided to the PSC algorithm and commands are generated on 20-s intervals. Taking advantage of the high frequency of measurements available, the filtered data was further refined and smoothed by using a windowed average. This is implemented with a trapezoidal integration approach. A windowing length was subjectively tuned such that the time-averaged value supplied as a measurement to the PSC algorithm is an average of the settled steady state response of engine rpm. An illustration of this windowing average is shown in Fig. (5).

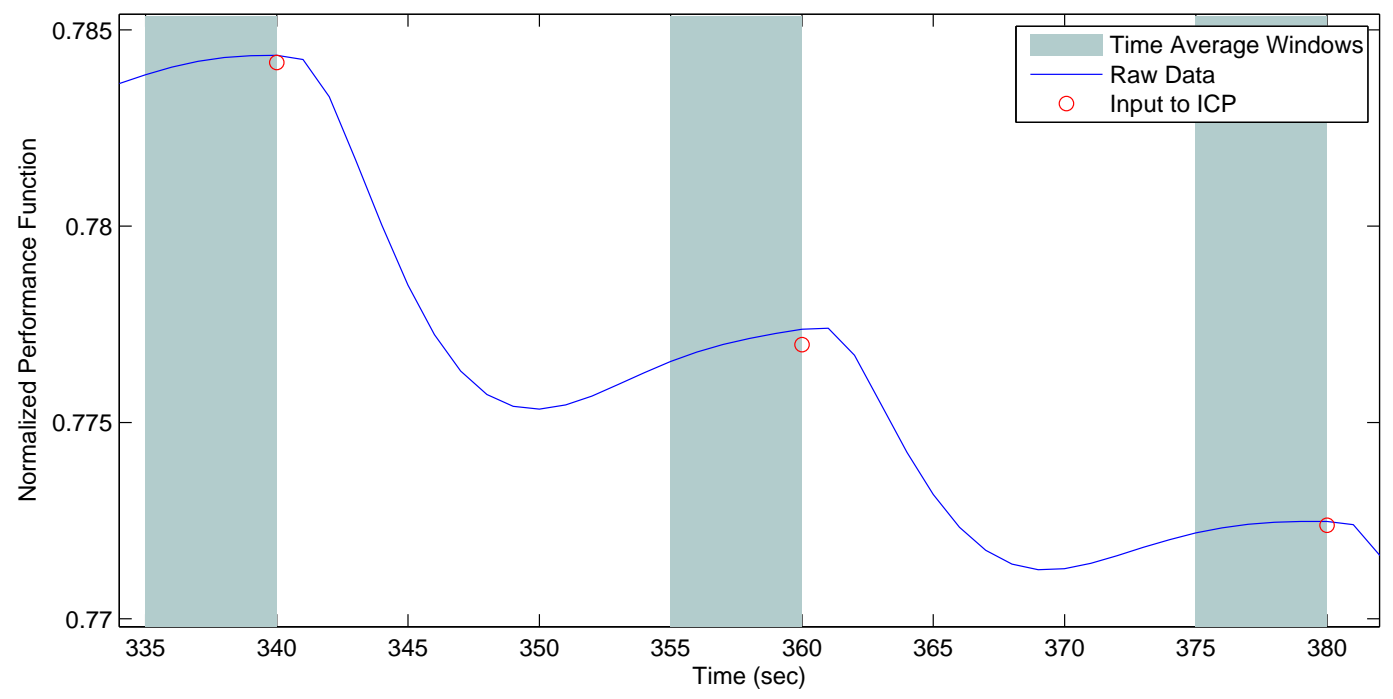

Figure 5. Time-averaging windows.

In addition to filtering the sensor data at the input of the PSC system, the output commands generated are also sent through first-order low-pass filters designed to prevent potentially large and abrupt step change surface commands which may lead to the vehicle moving off condition. This eases the workload of the autothrottle and auto-pilot controllers, but slows response time, leading to longer algorithm performance times. The filter was subjectively tuned with knowledge of this tradeoff.

\section{Simulation Results}

\section{A. Flight Condition}

A variety of flight conditions were analyzed in the simulation and a flight condition representative of a steady level cruise condition was chosen as an example for the results discussed below. The results shown are illustrative of the vehicle in a light weight, slats retracted, forward CG configuration and trimmed at a speed of 80 knots at an altitude of $5000 \mathrm{ft}$ MSL.

\section{B. Performance Function}

To prove algorithm convergence and analyze performance, the underlying performance function curve was generated by running the simulation with a range of clamshell positions and letting the vehicle trim to determine the performance function magnitude as a function of clamshell positions. This relationship was determined for the flight condition discussed above and is shown in Fig. (6). 


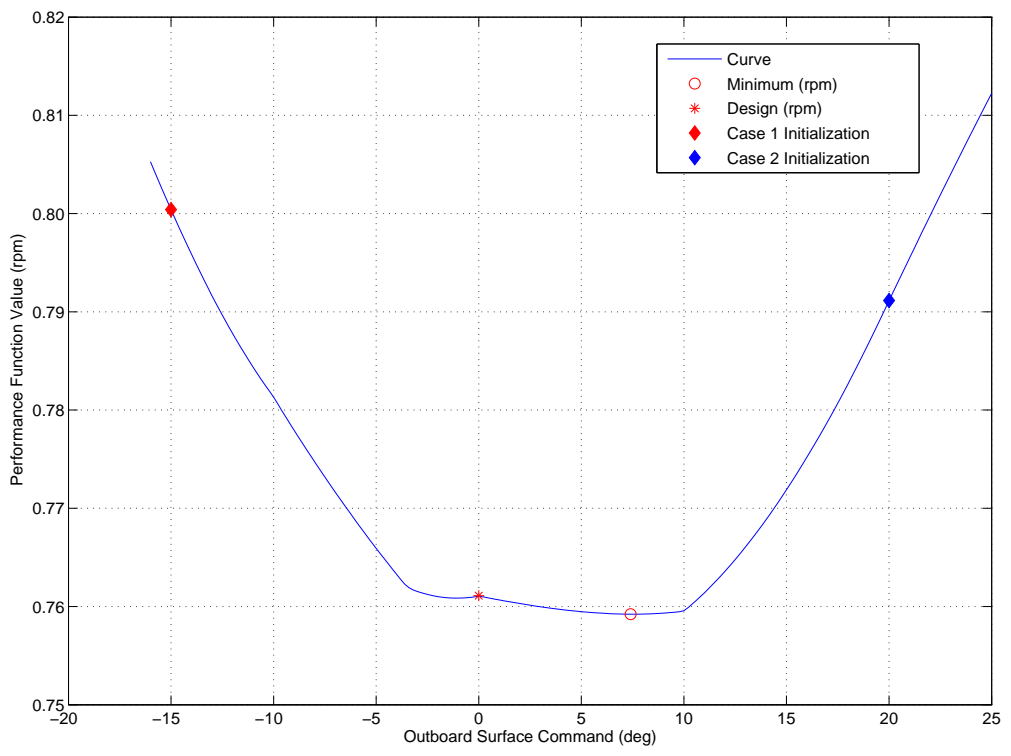

Figure 6. Performance function magnitude versus clamshell surface position.

\section{Algorithm Performance}

The algorithm was run from two initial conditions. Shown in Figs. (7) and (8) is the algorithm's performance for two cases. The average engine rpm is normalized by the engine rpm at 100 percent throttle setting. This was done to ease the development of the PSC system gains. The initialization for each case was chosen to show convergence when approaching from either side of the performance function minimum. These initialization clamshell surface defections are -15 and 20 deg for case 1 and case 2 respectively. The dotted line represents the time period during which the vehicle was allowed to re-trim at these initialization positions. The vehicle was allowed $200 \mathrm{~s}$ to re-trim at these new conditions before engaging the PSC algorithm. The configuration and tuning parameters used in these runs resulted in each run converging to approximately 7 deg. From the performance function curve shown in Fig. (6), the aero model reveals a local minimum very close to zero deg deflection. It should be noted that given a smaller value of the gradient gain multiplier in the ICP system, case 1 may converge to this location and not find the global minimum as shown in these results. This is expected, as the method simply tracks a gradient and is not a global search algorithm. This can be seen when performance in the presence of turbulence is discussed. Convergence time, ranging from approximately 3 to $7 \mathrm{~min}$, was primarily dictated by the closed-loop auto-pilot and auto-throttle system behavior influencing the performance function dynamics. 


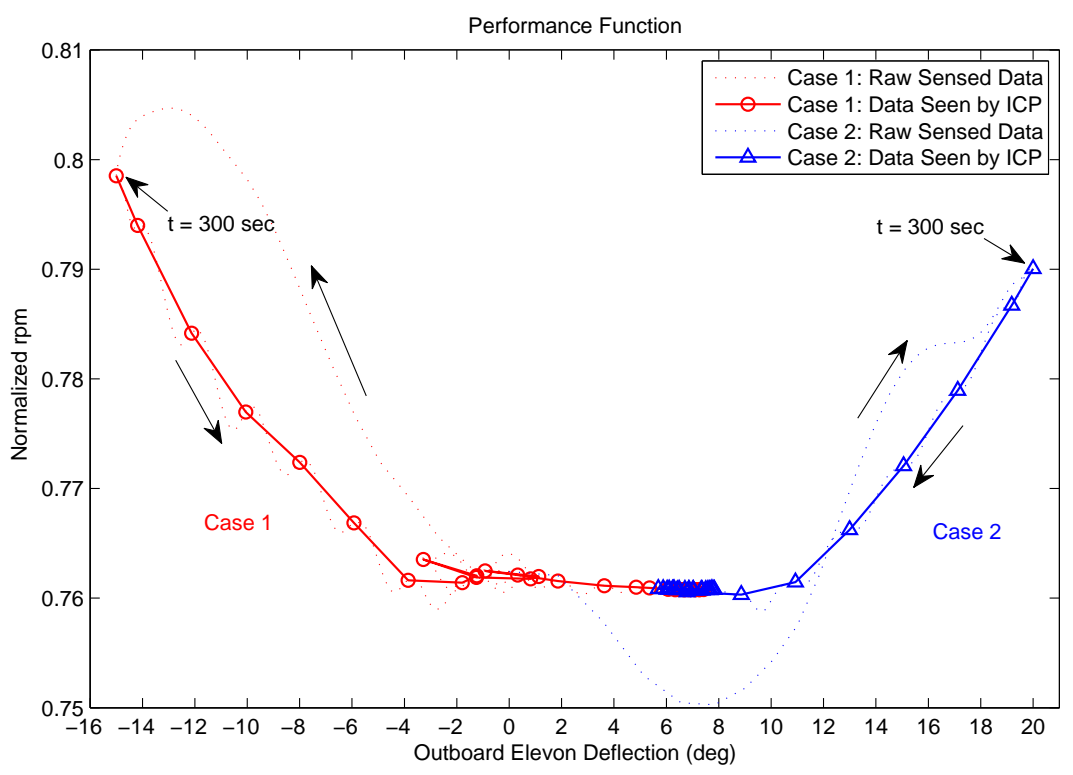

Figure 7. Algorithm performance function convergence.
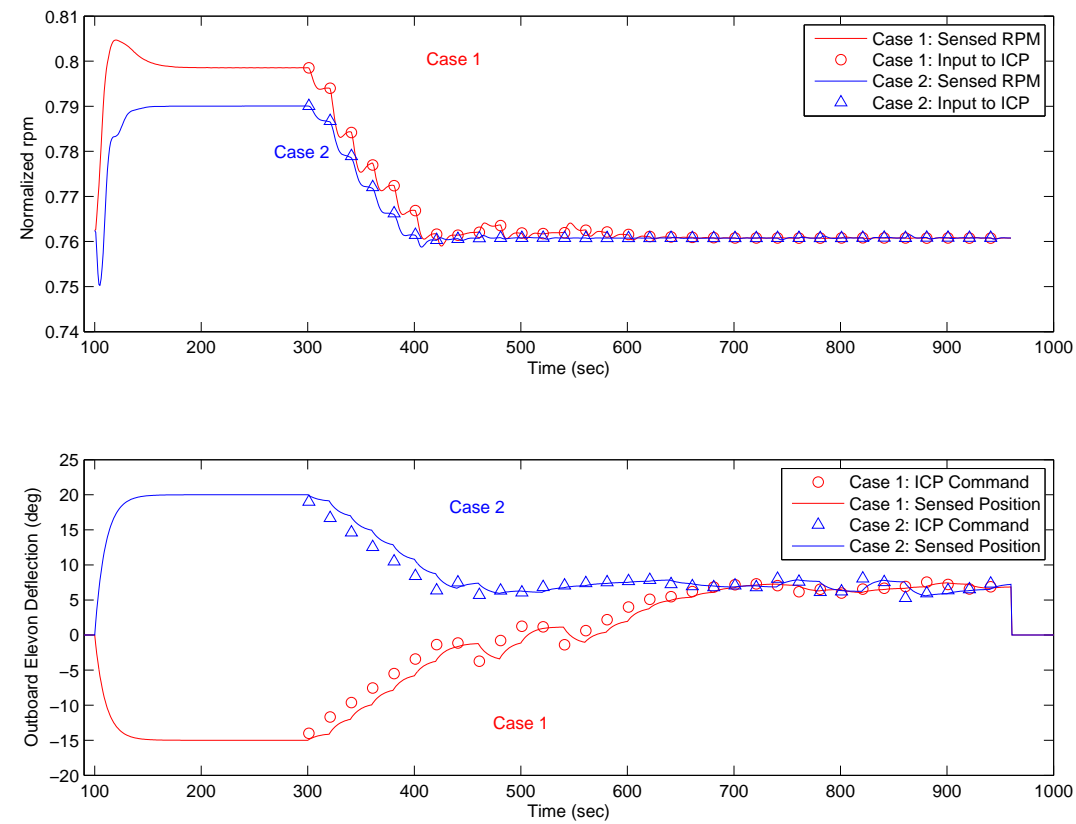

Figure 8. Algorithm time history convergence performance.

\section{Flight Feasibility Considerations}

Several problems arose during the implementation and the flight-test planning phase of the project which could possibly have hindered the flight-test feasibility of the PSC scheme on the X-48B platform. The performance function is very flat, and requires the ability to measure very small changes in performance. It is not expected that the system will improve performance dramatically within a short period of time, 
mainly due to the fact that the aircraft is already optimized by design. Instead, the system may gain small improvements accruing to significant performance gains over an extended period of time. Thus, sensors capable of measuring very small changes in response and actuators which respond to very small changes in commands are important for successful application of the intelligent control system. Topics identified are listed below:

- Actuator response

- Auto-throttle performance

- Turbulent conditions.

\section{A. Actuator Response}

The control surface actuators on the X-48B vehicle have a range of $70 \mathrm{deg}$, from 30 deg trailing-edge (TE) down deflection to approximately $40 \mathrm{deg} \mathrm{TE}$ up deflection. The actuators tend not to respond to command changes of less than approximately $0.5 \mathrm{deg}$. This creates oscillatory behavior around the desired extremum, never actually reaching the steady-state solution that maximizes performance. Additionally, the oscillatory behavior can increase fatigue load on the actuator system. The behavior of the sensed actuator position as well as a depiction of the system not responding to small changes in commands is shown in Fig. (9).

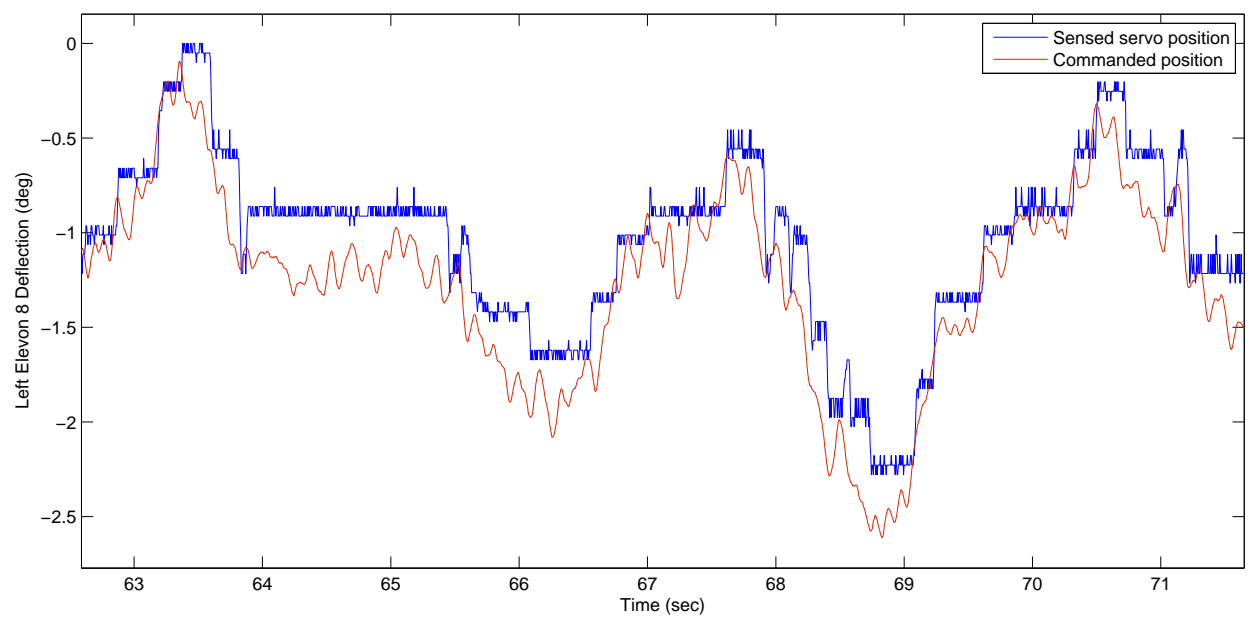

Figure 9. Actuator command versus measured response.

\section{B. Auto-Throttle Performance}

A small amount of flight data from previous flight-testing were available which included segments with the vehicle's auto-throttle system engaged, a condition necessary for the implementation. A small window of this data is shown in Fig. (10). During this segment, control surface activity is minimal, yet relatively large changes are seen in the engine rpm response. This behavior, given its magnitude, will have a corrupting impact on the algorithm and its ability to track the true optimal solution. Even when re-trimming the vehicle poorly before engaging the system, the total change in performance function is not significantly larger than this observed behavior. 

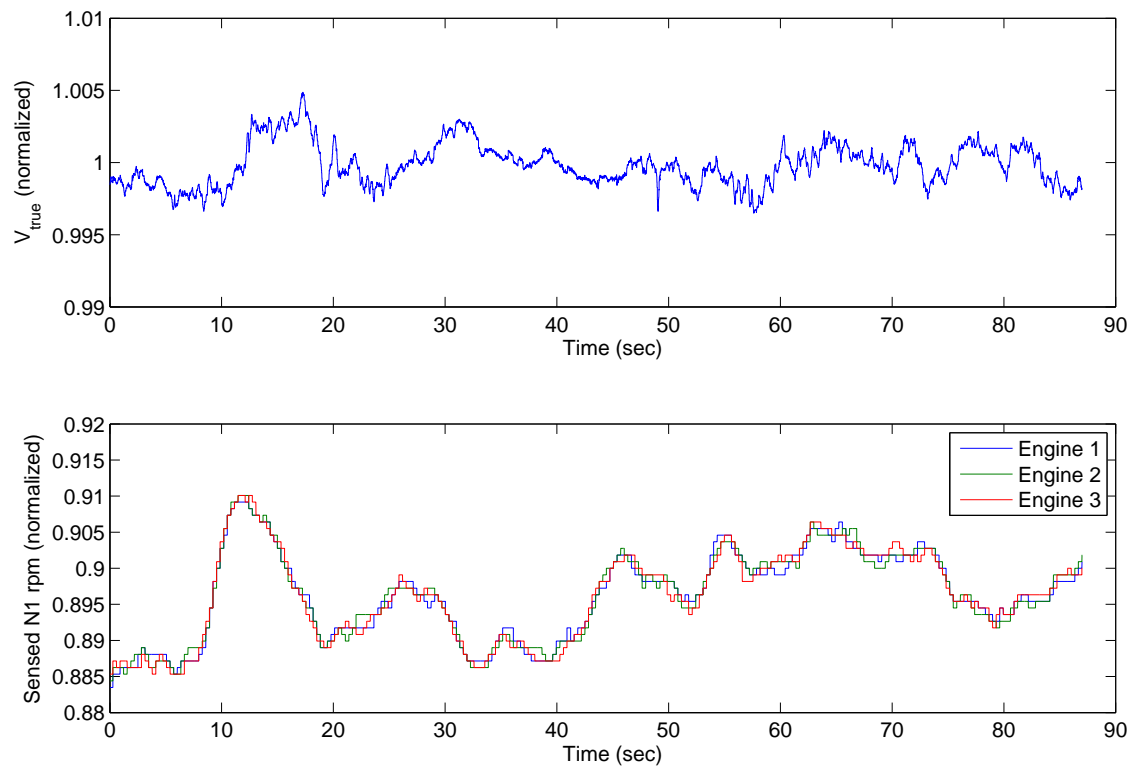

Figure 10. Auto-throttle response.

\section{Turbulent Conditions}

Simulated wind gusts act as disturbances to the intelligent control system. The DFRC gust model was implemented in the simulation and the two earlier cases were rerun. In the results shown, small gust magnitudes, less than 5 feet per second, were used. As expected, system performance degrades significantly in the presence of gusts. As seen in Figs. (11) and (12), the outboard clamshells are initialized to - 15 deg and matched the case 1 configuration. The system does move toward the minimum of the performance function, however, it does not reach the global optimal and instead oscillates between 0 and -6 deg deflection. This is around a performance function local minimum as seen in Fig. (6). This was the expected behavior.

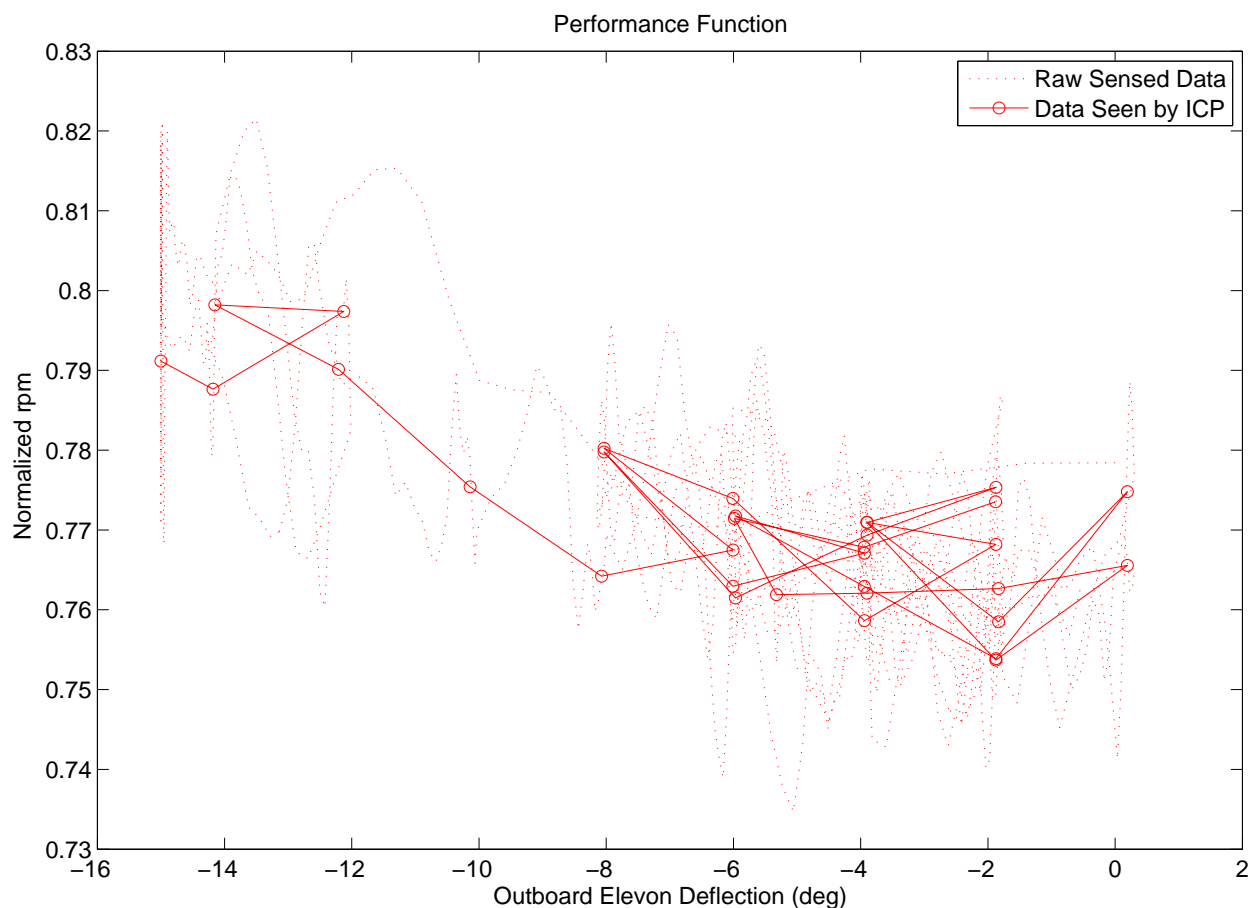

Figure 11. Performance function convergence in the presence of wind gusts. 

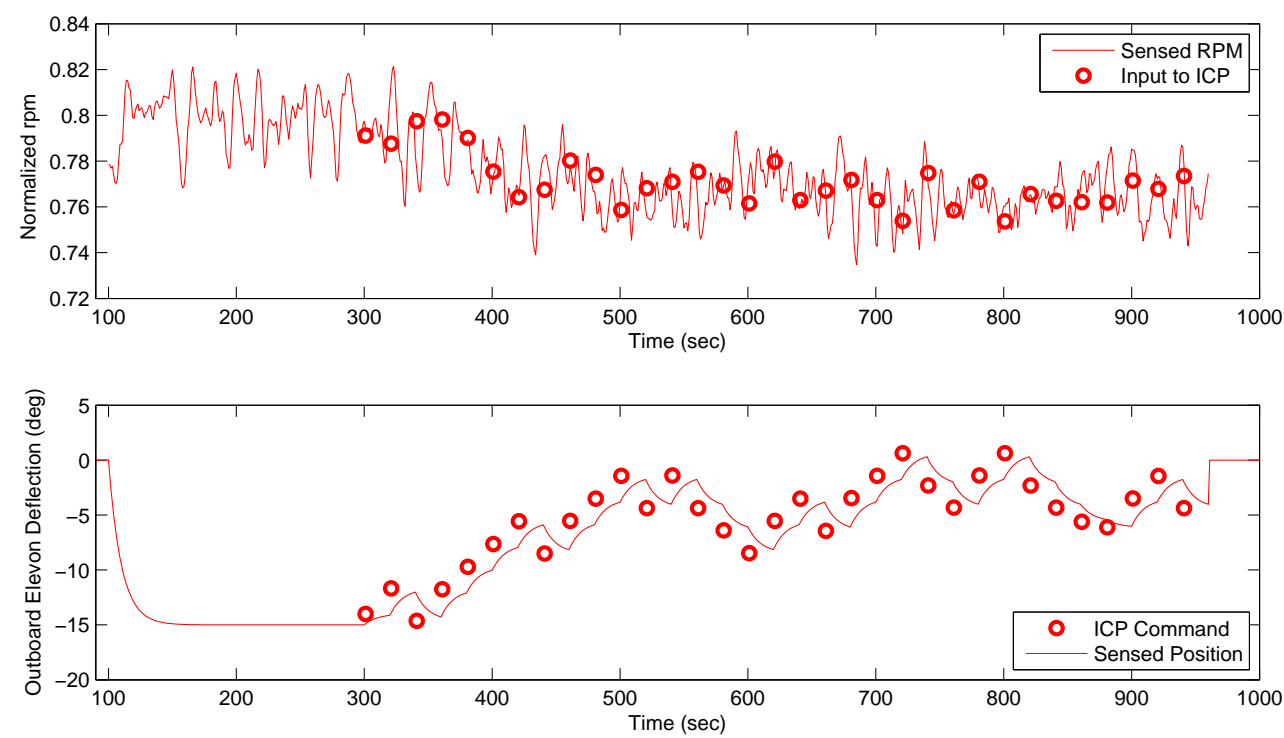

Figure 12. Time history convergence performance in the presence of wind gusts.

\section{Conclusions}

This paper focused on an intelligent control technology for drag reduction on the $\mathrm{X}-48 \mathrm{~B}$ vehicle. The system was demonstrated on the X-48B non-linear simulation. A peak-seeking control method integrated a time-varying Kalman filter for gradient estimation. To ease integration, a single-input single-output approach was chosen using steepest gradient descent. The framework, specific implementation considerations, simulation results, and flight feasibility issues related to this platform were discussed.

The algorithm performance was illustrated starting from initial conditions on both sides of the performance function. This algorithm proves to adequately track a minimum of a performance curve when given sufficient measurement information. In this application it is evident that even in the presence of small wind disturbances the algorithm tracks, however, performance benefits are reduced. This is not to imply that the algorithm cannot be successfully demonstrated during flight-testing, however the constraints enforced to simulate real conditions reduced the ability to demonstrate measurable improvements. Implemented on a vehicle not bounded by these constraints, adjustments could be made within the algorithm, that when averaged over many flight hours, would allow for measurable improvements.

\section{References}

\footnotetext{
${ }^{1}$ Collier, F., "Overview of NASA's Environmentally Responsible Aviation Project," 48th AIAA Aerospace Sciences Meeting, Orlando, Florida, January 42010.

${ }^{2}$ Liebeck, R. H., "Design of the Blended Wing Body Subsonic Transport," Journal of Aircraft, Vol. 41, No. 1, JanuaryFeburary 2004, pp. 21-22.

${ }^{3}$ Gilyard, G. B. and Orme, J. S., "Subsonic Flight Test Evaluation of a Performance Seeking Control Algorithm on an F-15 Airplane," NASA TM-4400, 1992.

${ }^{4}$ Orme, J. S. and Gilyard, G. B., "Preliminary Supersonic Flight Test Evaluation of Performance Seeking Control," NASA TM-4494, 1993.

${ }^{5}$ Gilyard, G. B. and Orme, J. S., "Performance Seeking Control: Program Overview and Future Directions," NASA TM-4531, 1992.

${ }^{6}$ Orme, J. S. and Conners, T. R., "Supersonic Flight Test Results of a Performance Seeking Control Algorithm on a NASA F-15 Aircraft," AIAA 94-3210, 1994.

${ }^{7}$ Orme, J. S. and Schkolnik, G. S., "Flight Assessment of the Onboard Propulsion System Model for the Performance Seeking Control Algorithm on an F-15 Aircraft," NASA TM-4705, 1995.

${ }^{8}$ Gilyard, G. B. and España, M., "On the Use of Controls for Subsonic Transport Performance Improvement: Overview and Future Directions," NASA TM-4605, 1994.
} 
${ }^{9}$ España, M. and Gilyard, G., "Direct Adaptive Performance Optimization of Subsonic Transports: A Periodic Perturbation Technique," NASA TM-4676, 1995.

${ }^{10}$ Gilyard, G., "Development of a Real-Time Transport Performance Optimization Methodology," NASA TM-4730, 1996.

${ }^{11}$ Gilyard, G., "In-Flight Transport Performance Optimization: An Experimental Flight Research Program and an Operational Scenario," NASA TM-97-206229, 1997.

${ }^{12}$ Gilyard, G. B., Georgie, J., and Barnicki, J. S., "Flight Test of an Adaptive Configuration Optimization System for Transport Aircraft," NASA TM-1999-206569, 1999.

${ }^{13}$ Ryan, J. J. and Speyer, J. L., "Peak-Seeking Control Using Gradient and Hessian Estimates," 2010 American Control Conference, Baltimore, MD, June 30 - July 02 2010, 978-1-4244-7427-1/10. 


\title{
Intelligent Control for Drag Reduction on the X-48B Vehicle
}

\author{
Brian Joseph Griffin*, Nelson A. Brown ${ }^{\dagger}$, and Seung Y. Yoo \\ NASA Dryden Flight Research Center, Edwards, California, 93523
}

\section{Presented by: Brian Griffin}

\footnotetext{
*Aerospace Engineer, Flight Controls and Dynamics Branch, P.O. Box 273, MS 4840D, brian.j.griffin@nasa.gov. ${ }^{\dagger}$ Aerospace Engineer, Flight Controls and Dynamics Branch, P.O. Box 273, MS 4840D, nelson.a.brown@nasa.gov.

$\ddagger$ Aerospace Engineer, Flight Controls and Dynamics Branch, P.O. Box 273, MS 4840D, seung.y.yoo@nasa.gov.
} 


\section{Topics Addressed}

- Vehicle Description

- Framework

$\checkmark$ Peak-Seeking Control

$\checkmark$ Time-varying Kalman filter

- Implementation

$\checkmark$ Allocation

$\checkmark \quad$ Filtering

- Simulation Results

- Flight Feasibility

$\checkmark$ Actuator Response

$\checkmark$ Auto-Throttle Performance

$\checkmark$ Turbulent Conditions 


\section{X-48B Brief Vehicle Description}

- $8.5 \%$ dynamically scaled

- $20.4 \mathrm{ft}$ wingspan and max take-off weight of $525 \mathrm{lb}$

- 20 control surfaces and two ground configurable slat positions

- 3 JetCat P200 engines capable of $54 \mathrm{lb}$ of thrust each
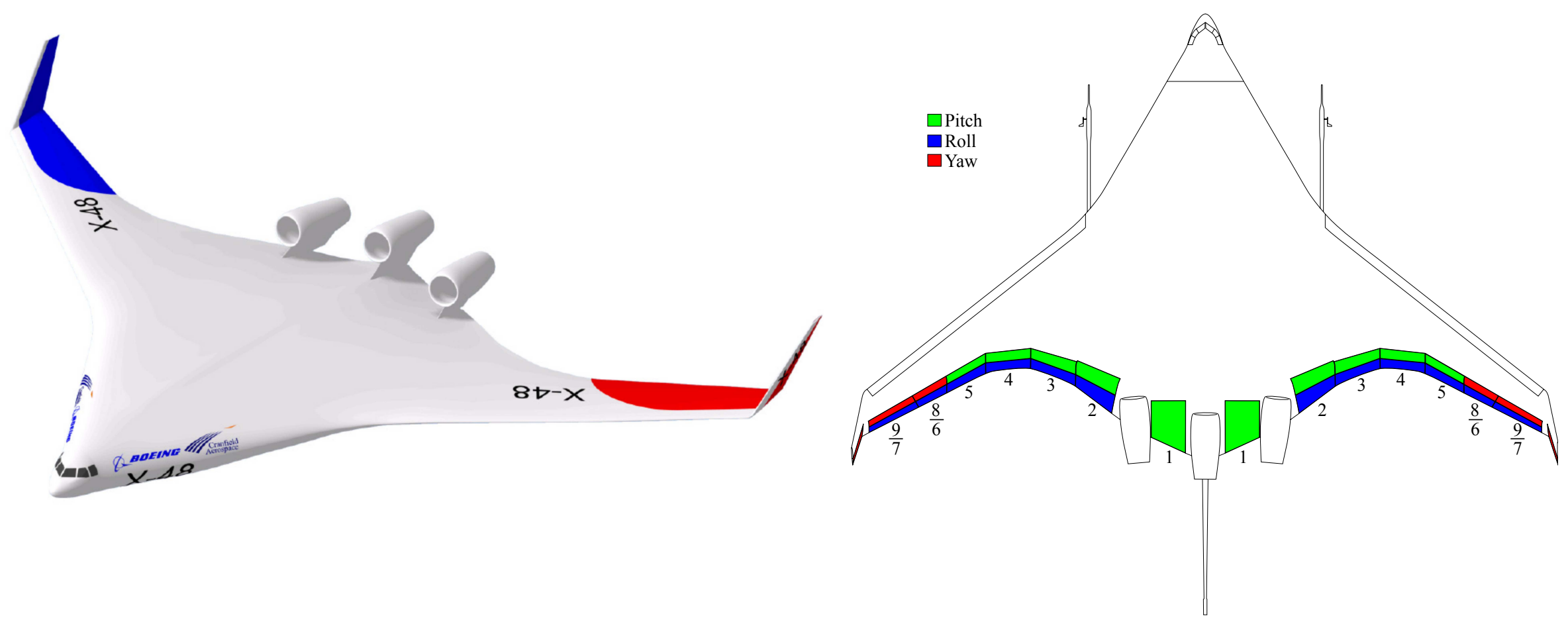

AIAA Guidance, Navigation, and Control Conference 


\section{Framework}

- Peak-Seeking Control Application

$\checkmark$ The goal of the intelligent control design using PSC is to control the plant in such a way as to ensure desired behavior in addition to driving some defined output, $x(t)$ of the plant $P$, to the extremum of a performance function, $f(x(t))$.

$\checkmark$ Outer-loop optimization.

$\checkmark$ Inner-loop control of plant is assumed.

$\checkmark$ Performance function defined in terms of simple independent parameter.

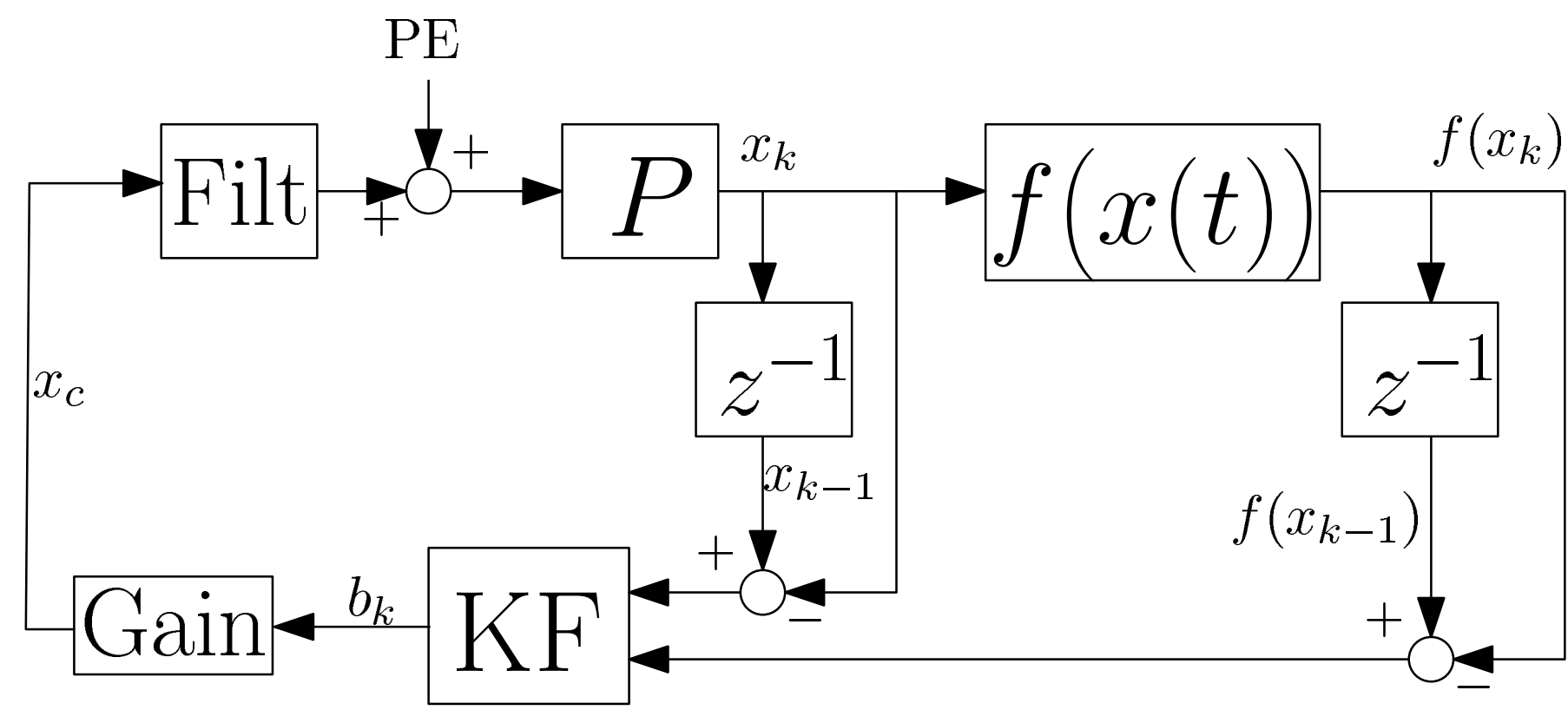




\section{Framework}

- Kalman Filter Implementation

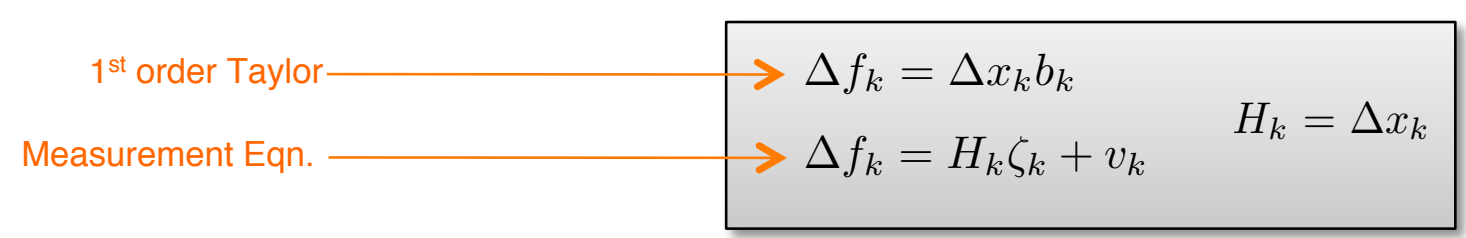

$v_{k}$ : zero-mean Gaussian white-noise with variance $V_{k}$

$$
\zeta_{k+1}=\zeta_{k}+w_{k} \lessdot \quad \text { Process Eqn. }
$$

$w_{k}$ : zero-mean Gaussian white-noise with variance $W_{k}$

$$
\begin{aligned}
K & =\hat{P}_{k} H_{k}^{\top}\left(H_{k} \hat{P}_{k} H_{k}^{\top}+V_{k}\right)^{-1} \\
\zeta_{k} & =\hat{\zeta}_{k}+K\left(\Delta f_{k}-H_{k} \hat{\zeta}_{k}\right) \\
P_{k} & =\left(I-K H_{k}\right) \hat{P}_{k} \\
\hat{\zeta}_{k+1} & =\zeta_{k} \\
\hat{P}_{k+1} & =P_{k}+W_{k}
\end{aligned}
$$

$P_{k}, \hat{P}_{(\cdot)}:$ current and predicted state covariance

$\zeta_{k}, \hat{\zeta}_{(\cdot)}$ : current and predicted KF state (PF gradient ) 


\section{X-48B Implementation}

- Peak-Seeking Control Dynamically Allocate Surfaces

$\checkmark$ Optimize span-wise lift distribution at a given flight condition.

$\checkmark$ Command identically sent to both upper an lower surfaces of all clamshell surfaces, on both sides of the vehicle.

$\checkmark$ Inner-loop control of plant removes other surfaces to maintain trim.

$\checkmark$ Engine-core speed (N2, rpm) averaged across 3 engines defines PF magnitude

$\checkmark$ At constant altitude and airspeed, a reduction in engine rpm is used to indicate a reduction in drag. 


\section{X-48B Allocation}

- Symmetric command to clamshell surface

$\checkmark$ Grouping clamshells (6-9) as one independent parameter keeps the implementation 1-dimensional.

$\checkmark$ Secondary approach including surface group (2-5) resulted in inner-loop fighting outer-loop PSC.

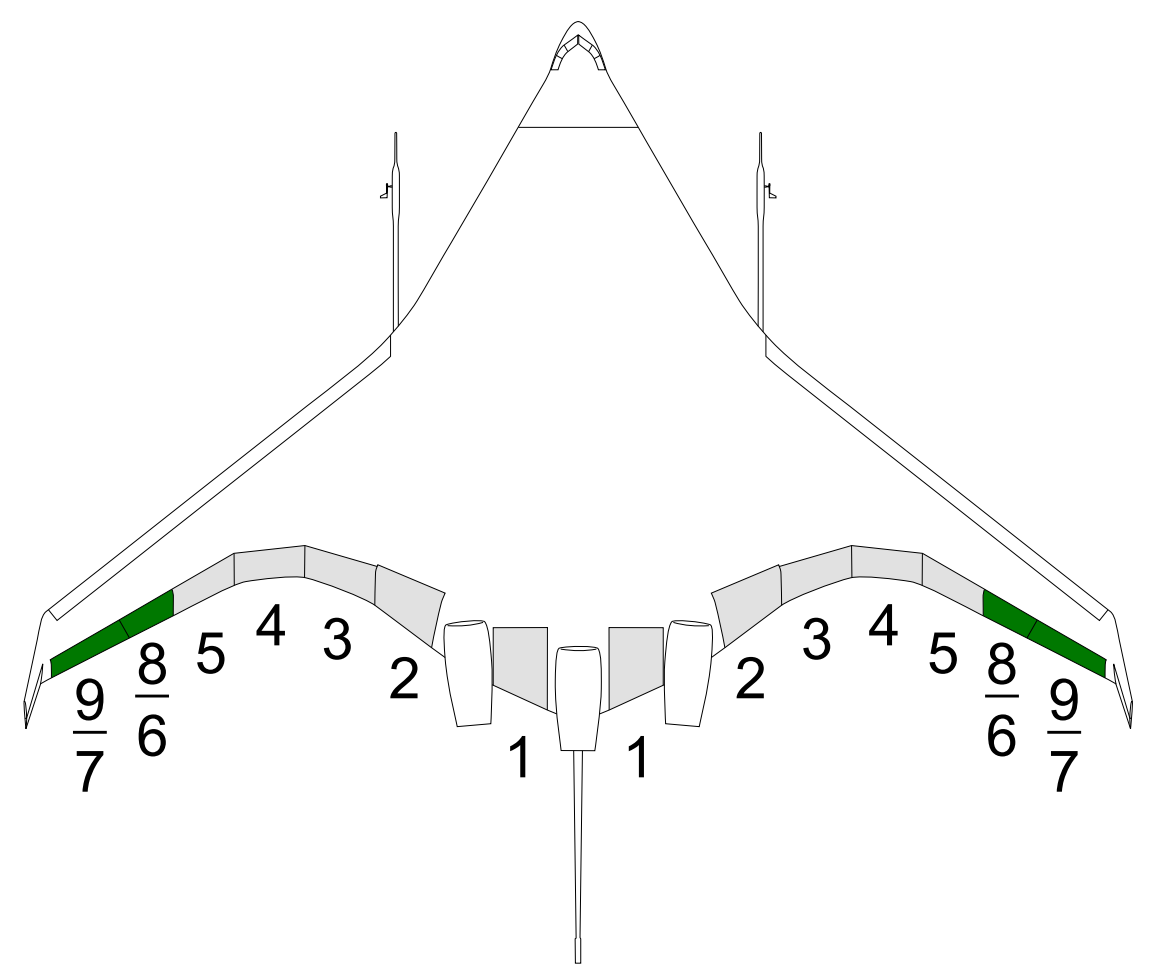




\section{X-48B Measurement / Command Filtering}

- Filtering and smoothing used at I/O plane of the PSC system

$\checkmark$ Outer-loop optimization ran much slower that inner-loop allowing averaging of many measurements can be used to supply the PSC algorithm.

$\checkmark$ Done using a subjectively tuned time-averaging window length.

$\checkmark$ Output commands filtered in addition to prevent abrupt step change surface commands.

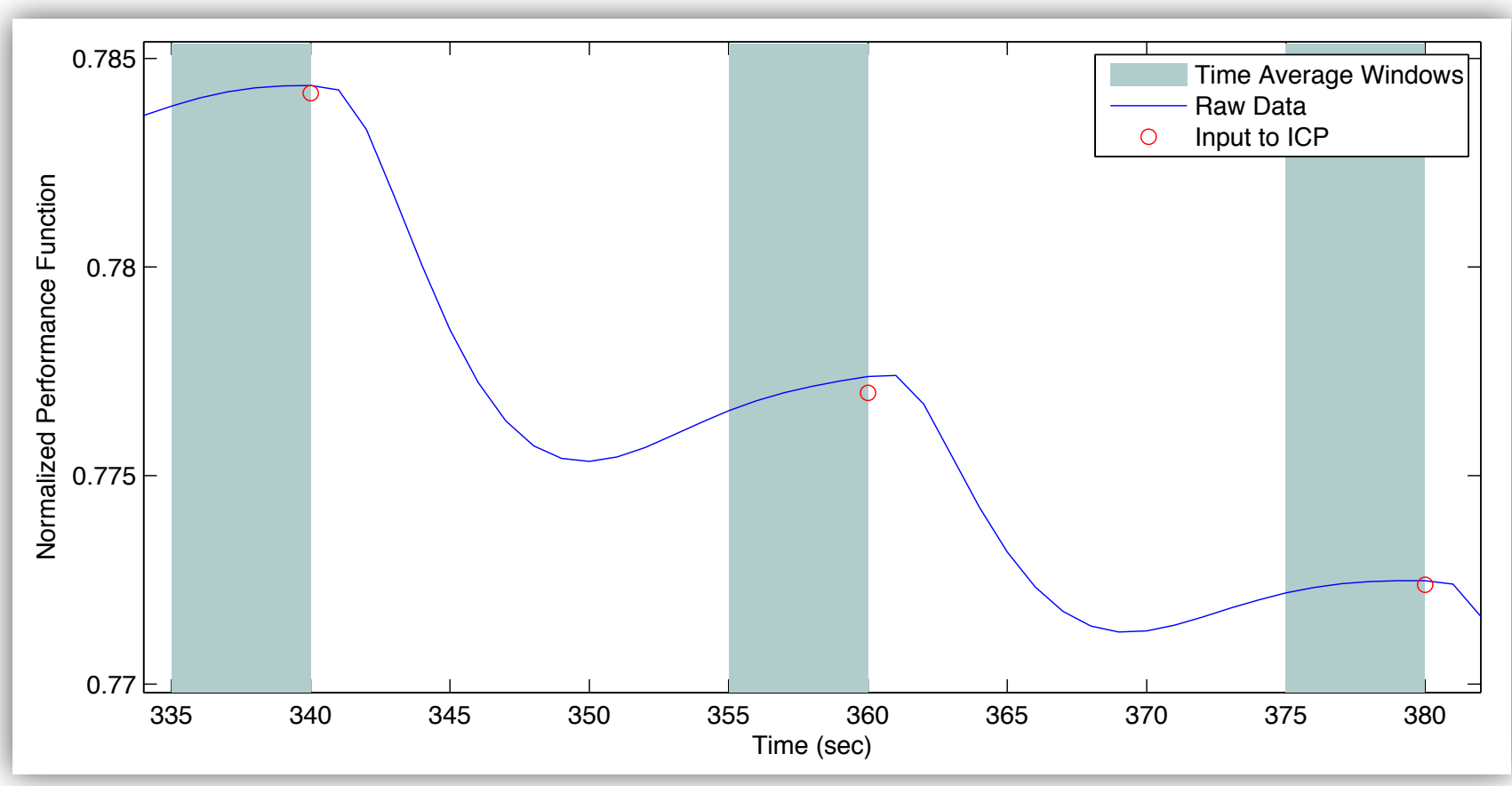




\section{Simulation Results}

- Flight Condition

$\checkmark$ Light weight, slat retracted, forward CG at 80 knots and $5000 \mathrm{ft} \mathrm{MSL}$

- Performance function

$\checkmark \quad$ PF magnitude as function of fixed clamshell surface position

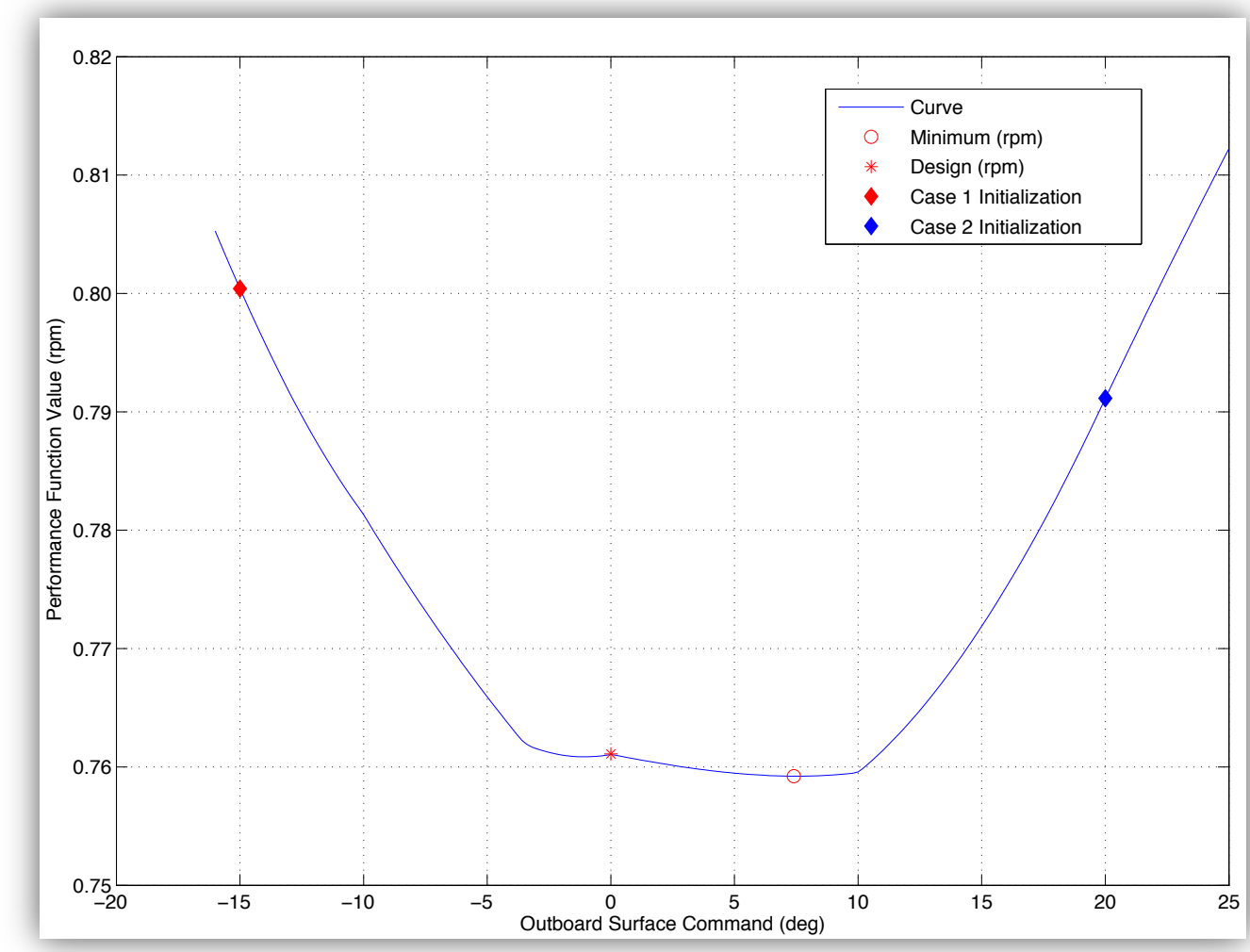




\section{Simulation Results}

- Algorithm Performance

$\checkmark$ Two initial conditions to show convergence from either side of the minimum

$\checkmark$ Vehicle allowed 200 seconds to re-trim before engagement
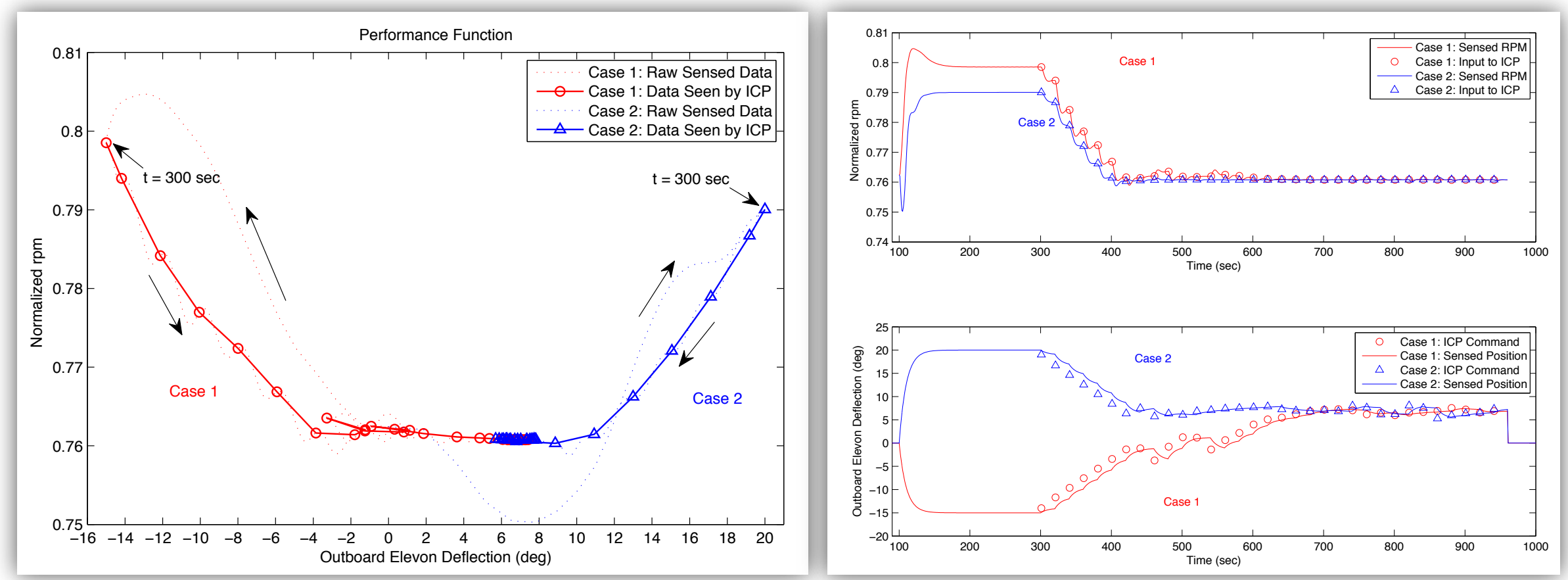


\section{Flight Feasibility Considerations}

- Flat performance function

- Improvements are expected to be small and would result in significant gains only over extended periods of time

$\checkmark$ Very sensitive sensors capable of measuring small changes in response

$\checkmark$ Actuators with ability to respond to minimal command changes required

- Issues Identified

$\checkmark$ Actuator response

$\checkmark$ Auto-throttle performance

$\checkmark$ Turbulent conditions 


\section{Flight Feasibility Considerations}

- Actuator Response

$\checkmark$ Actuators have a minimum change in command to which they will respond

$\checkmark$ Oscillatory behavior can result around a desired extremum increasing fatigue load and preventing the system from reaching steady-state solution

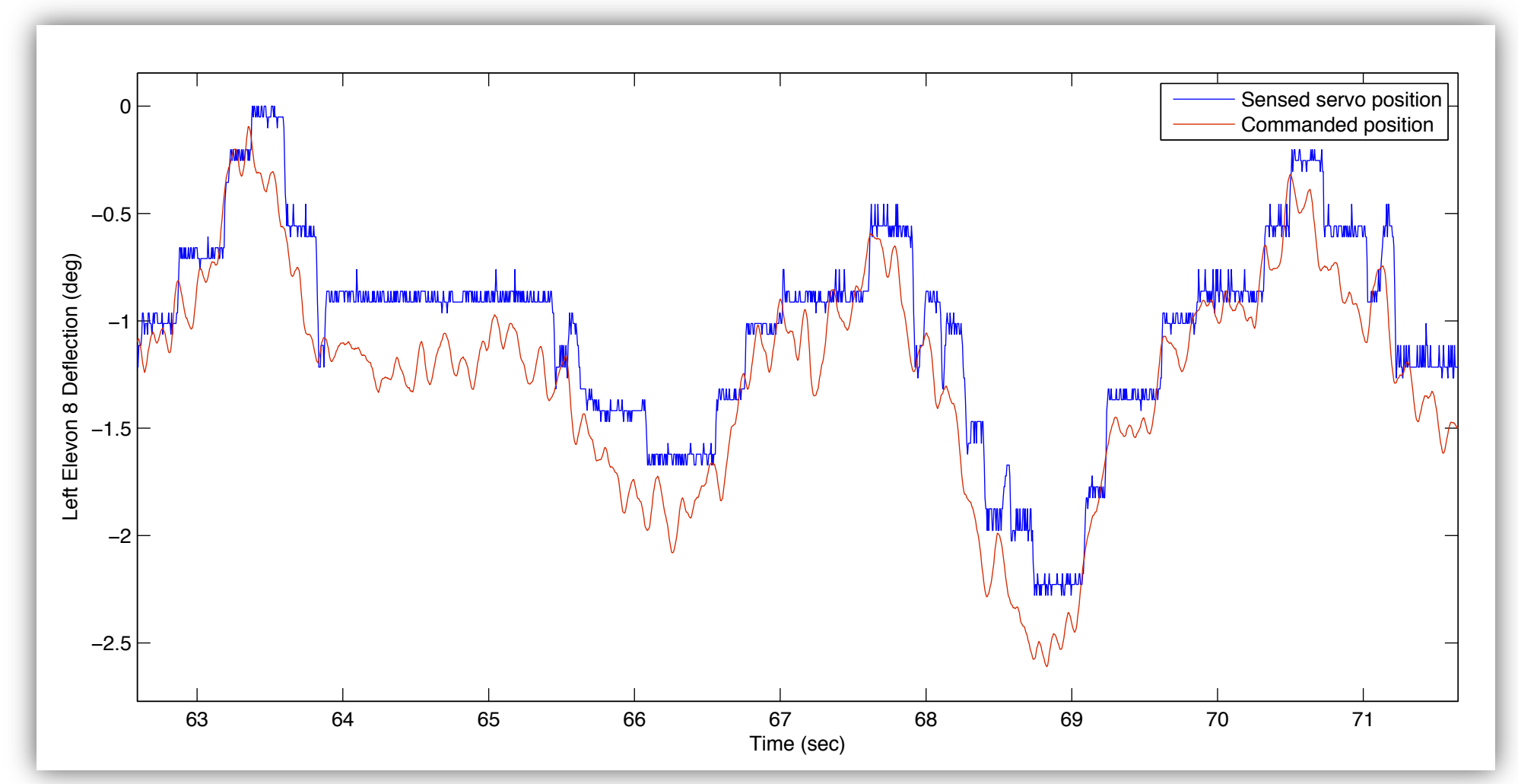




\section{Flight Feasibility Considerations}

- Auto-throttle performance

$\checkmark$ Auto-throttle response in flight during steady-state flight depicts substantial activity, reducing the ability to detect changes in performance due solely to the PSC algorithm commanded surface changes.

$\checkmark$ Behavior has a corrupting impact and seen as noise to the PSC system

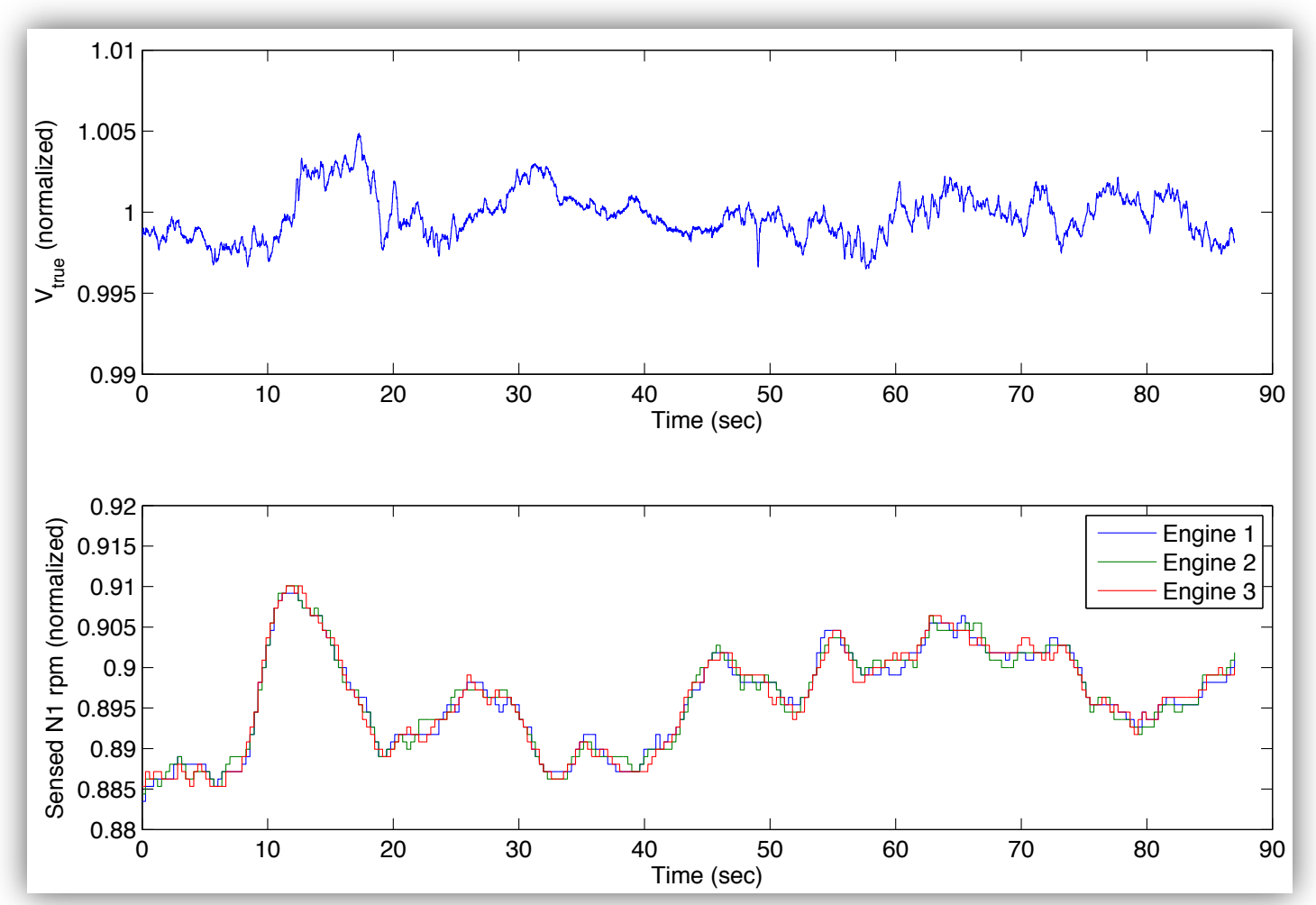




\section{Flight Feasibility Considerations}

- Turbulent Conditions

$\checkmark$ Simulated gusts act as disturbances to the PSC system

$\checkmark$ DFRC gust model implemented

$\checkmark$ Small gusts ( $<5 \mathrm{fps}$ ), significant degradations are seen, however, the system still tracks towards the minimum

$\checkmark$ Case 1 settings, namely same iteration length and filtering techniques
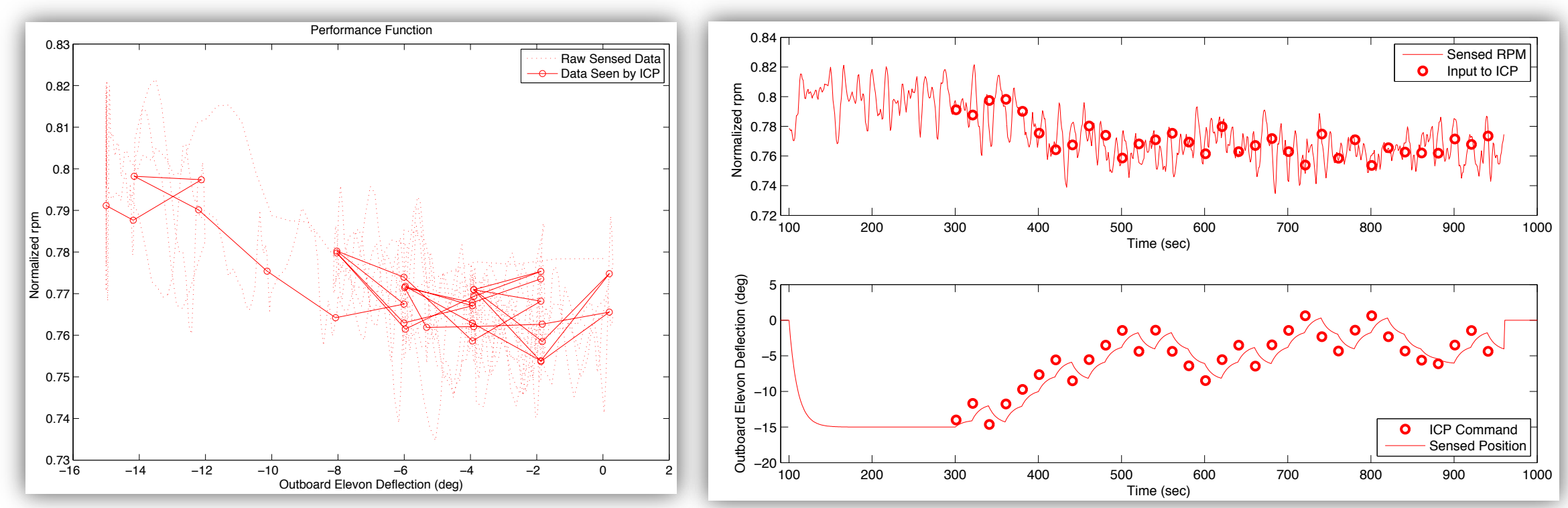


\section{Summary}

- PSC algorithm was successfully implemented and demonstrated on the full $\mathrm{X}-48 \mathrm{~B}$ non-linear simulation

- One dimensional approach taken to ease integration

- Algorithm adequately tracks a minimum of performance function given sufficient measurement information

- In presence of turbulence, in addition to other considerations, the performance is degraded and benefits reduced

- Does not imply that this cannot be flight-tested, however constraints enforced in this specific application reduce ability to demonstrate measurable improvements 


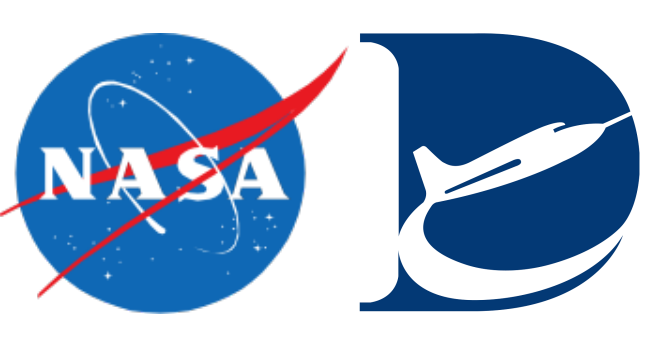

